\title{
The intergalactic medium in the cosmic web
}

\author{
Nicolas Tejos $\dagger$ \\ Department of Astronomy and Astrophysics, \\ UCO/Lick Observatory, University of California, \\ 1156 High Street, Santa Cruz, CA 95064, USA \\ email: ntejos@ucolick.org
}

\begin{abstract}
The intergalactic medium (IGM) accounts for $\gtrsim 90 \%$ of baryons at all epochs and yet its three dimensional distribution in the cosmic web remains mostly unknown. This is so because the only feasible way to observe the bulk of the IGM is through intervening absorption line systems in the spectra of bright background sources, which limits its characterization to being one-dimensional. Still, an averaged three dimensional picture can be obtained by combining and cross-matching multiple one-dimensional IGM information with three-dimensional galaxy surveys. Here, we present our recent and current efforts to map and characterize the IGM in the cosmic web using galaxies as tracers of the underlying mass distribution. In particular, we summarize our results on: (i) IGM around star-forming and non-star-forming galaxies; (ii) IGM within and around galaxy voids; and (iii) IGM in intercluster filaments. With these datasets, we can directly test the modern paradigm of structure formation and evolution of baryonic matter in the Universe.
\end{abstract}

Keywords. intergalactic medium; cosmology: large scale structure of the Universe; galaxies: formation; quasars: absorption lines

\section{Introduction}

The physics of the intergalactic medium (IGM) and its connection with galaxies are key to understanding the evolution of baryonic matter in the Universe. The IGM is the main reservoir of baryons at all epochs (e.g. Fukugita et al. 1998; Shull et al. 2012), and provides the primordial material for forming galaxies. Once galaxies are formed, supernovae (SNe) and active-galactic nuclei (AGN) feedback inject energy in the interstellar medium, some of which escapes the galaxies as winds, enriching the IGM with metals (e.g. Wiersma et al. 2011; Ford et al. 2014). Because of the continuous interplay between the IGM and galaxies, it is sensible (if not necessary) to study these two concepts simultaneously (e.g. Morris et al. 1993; Lanzetta et al. 1995; Tripp et al. 1998; Chen \& Mulchaey 2009; Prochaska et al. 2011; Tumlinson et al. 2011; Tejos et al. 2014; Werk et al. 2014).

The large scale environment in which matter resides also plays an important role. Given that baryonic matter is expected to fall into the considerably deeper gravitational potentials of dark matter, the IGM gas and galaxies should be predominantly found at such locations, forming the so-called 'cosmic web' (Bond et al. 1996). Galaxies appear to follow the filamentary structure which simulations predict (e.g. Springel et al. 2006), and their properties are partly shaped by environmental effects (e.g. Dressler 1980; Skibba et al. 2009). However, much less is known about the actual properties and distribution of the IGM in different cosmological environments. This is so because the only feasible way to observe the bulk of the IGM is through intervening absorption line systems in the spectra of bright background sources (e.g. quasi-stellar objects, gamma-ray bursts, galaxies), which limits its characterization to being one-dimensional.

$\dagger$ On behalf of our full collaboration. 

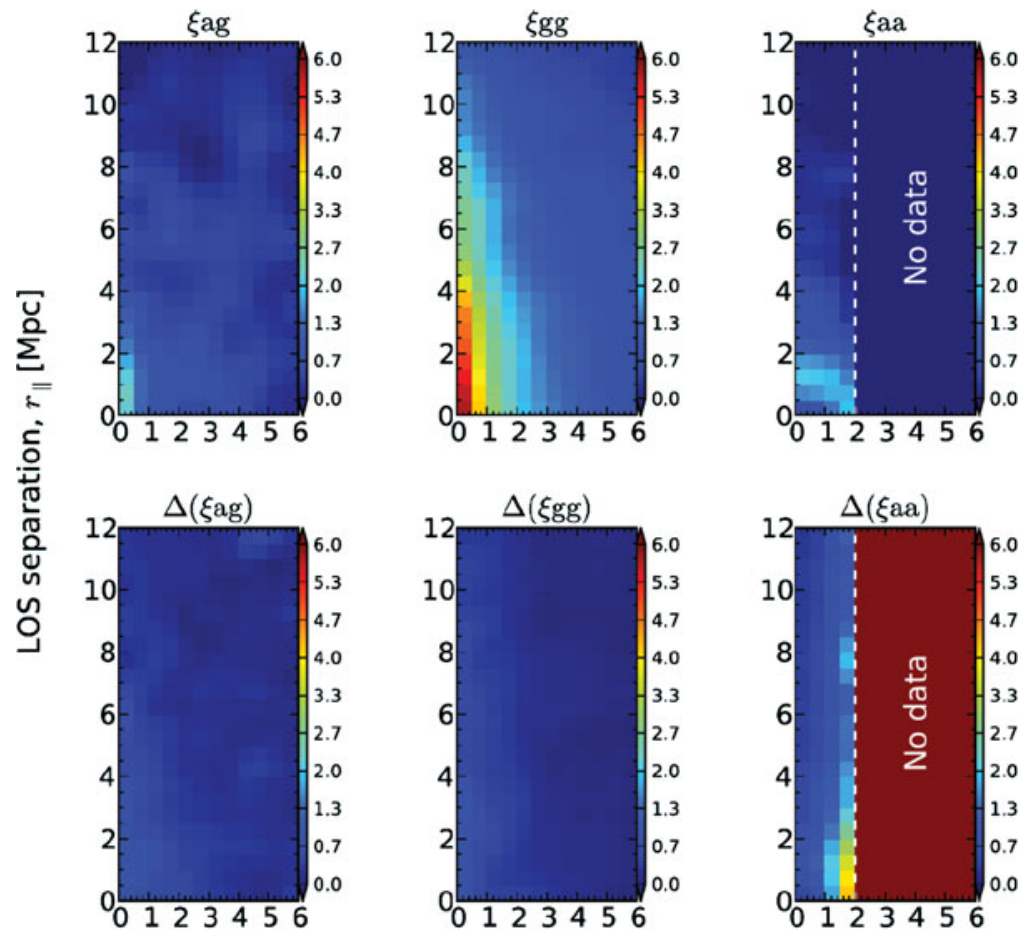

\section{Transverse separation, $r_{\perp}[\mathrm{Mpc}]$}

Figure 1. Two-dimensional two-point correlation functions (top panels) at $z \lesssim 1$, and their respective uncertainties (bottom panels). From left to right: H I-galaxy $\left(\xi_{\text {ag }}\right)$, galaxy-galaxy $\left(\xi_{\mathrm{gg}}\right)$ and $\mathrm{H} \mathrm{I}-\mathrm{H} \mathrm{I}\left(\xi_{\mathrm{aa}}\right)$ cross-correlations. Figure adapted from Tejos et al. (2014).

The advent of big galaxy surveys such as the 2dFGRS (Colless et al. 2001) or the SDSS (Abazajian et al. 2009), have revolutionized the study of the cosmic web and the largescale structure (LSS) of the Universe. This is eloquently demonstrated by the plethora of LSS catalogs that are currently available: from galaxy voids (e.g. Pan et al. 2012; Sutter et al. 2012; Nadathur \& Hotchkiss 2014; Way et al. 2014), galaxy filaments (e.g. Tempel et al. 2014), to galaxy groups and clusters (e.g. Hao et al. 2010; Rykoff et al. 2014). By combining and cross-matching multiple one-dimensional IGM information with galaxy and LSS surveys, an averaged three dimensional picture can be obtained. Here, we present our recent and current efforts to map and characterize the IGM in the cosmic web using galaxies as tracers of the underlying mass distribution.

\section{The IGM-galaxy cross-correlation}

The two-point correlation function between neutral hydrogen $(\mathrm{HI})$ and galaxies is a powerful statistical technique to assess the connection between the IGM and galaxies (e.g. Chen et al. 2005; Ryan-Weber 2006; Wilman et al. 2007; Chen \& Mulchaey 2009; Shone et al. 2010; Tejos et al. 2014).

In Tejos et al. (2014), we have recently published observational results on the $\mathrm{H}$ Igalaxy two-point cross-correlation at $z \lesssim 1$ ( $\xi_{\text {ag }}$; see Fig. 1$)$. These results come from the largest sample ever done for such an analysis, comprising about $\sim 700 \mathrm{H} \mathrm{I}$ absorption line systems in the UV spectra of 8 background QSOs, in 6 different fields observed with the HST, and about $\sim 17000$ galaxies with spectroscopic redshifts around these QSOs 
sightlines, coming from our own spectroscopic surveys, and previously published catalogs by the VVDS (Le Fèvre et al. 2013) and GDDS (Abraham et al. 2004) galaxy surveys.

Apart from $\xi_{\text {ag }}$, we also measured the $\mathrm{H} \mathrm{I}-\mathrm{H}_{\mathrm{I}}\left(\xi_{\text {aa }}\right)$ and galaxy-galaxy $\left(\xi_{\mathrm{gg}}\right)$ two-point auto-correlations. Our survey is one of the few in which these three quantities have been measured from the same dataset, and independently from each other. Comparing the results from $\xi_{\text {ag }}, \xi_{\text {aa }}$ and $\xi_{\text {gg }}$, we constrained the IGM-galaxy statistical connection, as a function of both $\mathrm{H}$ I column density and galaxy star formation activity, on $\sim 0.5-10 \mathrm{Mpc}$ scales. Our results are consistent with the following conclusions: (i) the bulk of $\mathrm{H}$ I systems on $\sim$ Mpc scales have little velocity dispersion $\left(\lesssim 120 \mathrm{~km} \mathrm{~s}^{-1}\right)$ with respect to the bulk of galaxies (i.e. no strong galaxy outflow/inflow signal is detected); (ii) the vast majority ( 100\%) of $\mathrm{H}$ I systems with $N_{\mathrm{HI}}>10^{14} \mathrm{~cm}^{-2}$ and star-forming galaxies are distributed in the same locations, together with $75 \pm 15 \%$ of non-star forming galaxies; (iii) $25 \pm 15 \%$ of non-star-forming galaxies reside in galaxy clusters and are not correlated with $\mathrm{H}$ I systems at scales $\lesssim 2 \mathrm{Mpc}$; and (iv) $>50 \%$ of $\mathrm{H}$ I systems with $N_{\mathrm{HI}}<10^{14} \mathrm{~cm}^{-2}$ reside within galaxy voids and hence are not correlated with luminous galaxies.

\section{The IGM within and around galaxy voids}

In Tejos et al. (2012) we have recently measured the properties of H I absorption line systems within and around galaxy voids at $z \leqslant 0.1$, using the galaxy void catalog published by Pan et al. (2011) and the low- $z$ H I absorption line catalog published by Danforth \& Shull (2008). Our key findings can be summarized as follows: (i) there is a significant excess of IGM gas at the edges of galaxy voids with respect to the random expectation, consistent with the overdensity of galaxies defining such voids; and (ii) inside galaxy voids the IGM gas matches the random expectation, inconsistent with the underdensity of galaxies defining such voids. In other words, there were no apparent IGM voids detected at the positions of galaxy voids.

We also showed that the column density $\left(N_{\mathrm{HI}}\right)$ and Doppler parameter $\left(b_{\mathrm{HI}}\right)$ distributions of $\mathrm{H}$ I lines inside and outside galaxy voids were not remarkably different, with only a $\sim 95 \%$ and $\sim 90 \%$ probability of rejecting the null-hypothesis of both samples coming from the same parent population, respectively. Still, a trend was present, in which galaxy void absorbers have systematically lower values of both $N_{\mathrm{HI}}$ and $b_{\mathrm{HI}}$ than those found outside galaxy voids. By performing a similar analysis using a state-of-the-art hydrodynamical cosmological simulation (GIMIC; Crain et al. 2009), we showed that these observed trends are qualitatively consistent with current theoretical expectations. However, more quantitative comparisons of the gas properties in galaxy voids between hydrodynamical simulations tuned to explore low density environments (e.g. Ricciardelli et al. 2013) and observations (e.g. Tejos et al. 2012), are required.

\section{The IGM in intercluster filaments}

Galaxy clusters represent the densest nodes in the cosmic web (with dark matter halo masses of $M \gtrsim 10^{14} M_{\odot}$ ) and as such, $N$-body numerical simulations predict a high probability of finding intercluster filaments between galaxy cluster pairs when separated by $\lesssim 10-20 \mathrm{Mpc}$ (e.g. Colberg et al. 2005; González \& Padilla 2010). Hydrodynamical simulations predict that an important fraction of baryons at low- $z$ are in a diffuse, shock heated gas phase with $T \sim 10^{5}-10^{6} \mathrm{~K}$ in these dense filaments, commonly referred to as the warm-hot intergalactic medium (WHIM; Cen \& Ostriker 1999; Davé et al. 2001). However, this WHIM has been very elusive and difficult to observe (e.g. Richter et al. 2006). 
Here, we have presented preliminary results on the properties of the IGM in intercluster filaments at $0.1 \leqslant z \leqslant 0.47$ by using a single QSO observed with the HST/COS UV spectrograph, whose sightline intersects 7 independent cluster-pairs at impact parameters $<5 \mathrm{Mpc}$ from the intercluster axes. This technique allowed us to perform, for the first time, a systematic and statistical measurement of the incidence of $\mathrm{H} \mathrm{I}$ and $\mathrm{O}$ VI absorption lines associated to intercluster filaments. We constrained the geometry and physical properties of the IGM gas lying between clusters. Our results are consistent with a filamentary geometry for the gas, and the presence of both broad $\mathrm{HI}\left(>50 \mathrm{~km} \mathrm{~s}^{-1}\right)$ and $\mathrm{O}$ vi hint towards the existence of a WHIM (Tejos et al. in prep.).

This work was partly funded by CONICYT/PFCHA 72090883 (Chile).

\section{References}

Abraham, R. G., Glazebrook, K., McCarthy, P. J., et al. 2004, AJ, 127, 2455

Abazajian, K. N., Adelman-McCarthy, J. K., Agüeros, M. A., et al. 2009, ApJS, 182, 543

Bond, J. R., Kofman, L., \& Pogosyan, D. 1996, Nature, 380, 603

Cen, R. \& Ostriker, J. P. 1999, ApJ, 514, 1

Chen, H.-W., Lanzetta, K. M., Webb, J. K., \& Barcons, X. 1998, ApJ, 498, 77

Chen, H.-W., Prochaska, J. X., Weiner, B. J., et al. 2005, ApJ(Letters), 629, L25

Chen, H.-W. \& Mulchaey, J. S. 2009, ApJ, 701, 1219

Colberg, J. M., Krughoff, K. S., \& Connolly, A. J. 2005, MNRAS, 359, 272

Colless, M., Dalton, G., Maddox, S., et al. 2001, MNRAS, 328, 1039

Crain, R. A., Theuns, T., Dalla Vecchia, C., et al. 2009, MNRAS, 399, 1773

Danforth, C. W. \& Shull, J. M. 2008, ApJ, 679, 194

Davé, R., Cen, R., Ostriker, J. P., et al. 2001, ApJ, 552, 473

Dressler, A. 1980, ApJ, 236, 351

Ford, A. B., Davé, R., Oppenheimer, B. D., et al. 2014, MNRAS, 444, 1260

Fukugita, M., Hogan, C. J., \& Peebles, P. J. E. 1998, ApJ, 503, 518

González, R. E. \& Padilla, N. D. 2010, MNRAS, 407, 1449

Hao, J., McKay, T. A., Koester, B. P., et al. 2010, ApJS, 191, 254

Lanzetta, K. M., Bowen, D. V., Tytler, D., \& Webb, J. K. 1995, ApJ, 442, 538

Le Fèvre, O., Cassata, P., Cucciati, O., et al. 2013, A\& $A$, 559, A14

Morris, S. L., Weymann, R. J., Dressler, A., et al. 1993, ApJ, 419, 524

Nadathur, S. \& Hotchkiss, S. 2014, MNRAS, 440, 1248

Pan, D. C., Vogeley, M. S., Hoyle, F., Choi, Y.-Y., \& Park, C. 2012, MNRAS, 421, 926

Prochaska, J. X., Weiner, B., Chen, H.-W., Mulchaey, J., \& Cooksey, K. 2011, ApJ, 740, 91

Ricciardelli, E., Quilis, V., \& Planelles, S. 2013, MNRAS, 434, 1192

Richter, P., Savage, B. D., Sembach, K. R., \& Tripp, T. M. 2006, A\&A, 445, 827

Ryan-Weber, E. V. 2006, MNRAS, 367, 1251

Rykoff, E. S., Rozo, E., Busha, M. T., et al. 2014, ApJ, 785, 104

Shone, A. M., Morris, S. L., Crighton, N., \& Wilman, R. J. 2010, MNRAS, 402, 2520

Shull, J. M., Smith, B. D., \& Danforth, C. W. 2012, ApJ, 759, 23

Skibba, R. A., Bamford, S. P., Nichol, R. C., et al. 2009, MNRAS, 399, 966

Springel, V., Frenk, C. S., \& White, S. D. M. 2006, Nature, 440, 1137

Sutter, P. M., Lavaux, G., Wandelt, B. D., \& Weinberg, D. H. 2012, ApJ, 761, 44

Tejos, N., Morris, S. L., Crighton, N. H. M., et al. 2012, MNRAS, 425, 245

Tejos, N., Morris, S. L., Finn, C. W., et al. 2014, MNRAS, 437, 2017

Tempel, E., Stoica, R. S., Martínez, V. J., et al. 2014, MNRAS, 438, 3465

Tripp, T. M., Lu, L., \& Savage, B. D. 1998, ApJ, 508, 200

Tumlinson, J., Thom, C., Werk, J. K., et al. 2011, Science, 334, 948

Way, M. J., Gazis, P. R., \& Scargle, J. D. 2014, arXiv:1406.6111

Werk, J. K., Prochaska, J. X., Tumlinson, J., et al. 2014, ApJ, 792, 8

Wiersma, R. P. C., Schaye, J., \& Theuns, T. 2011, MNRAS, 415, 353

Wilman, R. J., Morris, S. L., Jannuzi, B. T., Davé, R., \& Shone, A. M. 2007, MNRAS, 375, 735 